\title{
OPEN Regional homogeneity abnormalities of resting state brain activities in children with growth hormone deficiency
}

\author{
Fanyu Zhang ${ }^{1,2}$, Bo Hua ${ }^{1,2}$, Mei Wang ${ }^{3}$, Tengfei Wang ${ }^{1,2}$, Zhongxiang Ding ${ }^{3 凶} \&$ \\ Ju-Rong Ding ${ }^{1,2 \bowtie}$
}

Growth hormone deficiency (GHD) is a common developmental disorder in children characterized by low levels of growth hormone secretion, short stature, and multiple cognitive and behavioral problems, including hyperactivity, anxiety, and depression. However, the pathophysiology of this disorder remains unclear. In order to investigate abnormalities of brain functioning in children with GHD, we preformed functional magnetic resonance imaging and regional homogeneity (ReHo) analysis in 26 children with GHD and 15 age- and sex-matched healthy controls (HCs) in a resting state. Compared with HCs, children with GHD exhibited increased ReHo in the left putamen and decreased ReHo in the right precentral gyrus, reflecting a dysfunction of inhibitory control. Decreased ReHo was also identified in the orbital parts of the bilateral superior frontal gyrus and the medial part of the left superior frontal gyrus, a finding that correlated with the inappropriate anxiety and depression that are observed in this patient population. Our results provide imaging evidence of potential pathophysiologic mechanisms for the cognitive and behavioral abnormalities of children with GHD.

Short stature, a prevalent disease in the pediatric endocrine clinic, is characterized by an annual growth in height of less than $5 \mathrm{~cm}$ and a height greater than two standard deviations below the average for normal children of the same race, sex, and age ${ }^{1,2}$. Short stature may either be idiopathic or caused by growth hormone deficiency (GHD). Idiopathic short stature (ISS) is the most common type of short stature, which refers to short stature without underlying pathological causes ${ }^{3}$. GHD is one of the most common causes of pathological short stature ${ }^{4}$. Studies using clinical psychological evaluations have shown greater social, thought, and attention problems, and greater delinquent behavior in children with GHD than in healthy controls (HCs) ${ }^{5}$. In addition, a large number of magnetic resonance imaging (MRI) studies have shown that patients with GHD have structural abnormalities in the brain, including an interrupted pituitary stalk and a hypoplastic hypophysis ${ }^{6,7}$, as well as an ectopically located posterior pituitary gland ${ }^{8}$.

Functional magnetic resonance imaging (fMRI) reflects changes in activity intensity in brain regions by detecting changes in the amount of oxygen carried by hemoglobin ${ }^{9,10}$ As a result, the fMRI signal is often referred to as the blood oxygen level-dependent (BOLD) signal ${ }^{11}$. Recently, resting-state fMRI (rs-fMRI) techniques have been used widely in studies of brain activity in various neuropsychiatric disorders, including attentiondeficit/hyperactivity disorder ${ }^{12}$, social anxiety disorder ${ }^{13}$, mesial temporal lobe epilepsy ${ }^{14}$, and major depressive disorder ${ }^{15}$. An existing rs-fMRI study has shown that compared with children with ISS, children with GHD have lower functional connection density in the left postcentral gyrus, right precentral gyrus, and Brodmann's area of left cerebellar lobules $7 \mathrm{~b}$ and 6 , as well as lower functional connectivity of the brain network involving the posterior cerebellar lobes and sensorimotor network ${ }^{16}$. However, at present, there is no information about the activity within the brain clusters of children with GHD, especially regarding the consistency of activities.

Regional homogeneity $(\mathrm{ReHo})^{17}$ is a method to measure the similarity of a voxel's activity-time series within a given cluster. It assumes the spontaneous BOLD signal's change of voxels within clusters are synchronized and can be modulated during cognitive tasks; therefore, it is suitable for analyzing rs-fMRI data ${ }^{17}$. Abnormalities of ReHo may reflect disorders of local brain functioning ${ }^{18}$ and may indicate abnormal mental activity and

\footnotetext{
${ }^{1}$ Artificial Intelligence Key Laboratory of Sichuan Province, Sichuan University of Science and Engineering, Zigong, China. ${ }^{2} S c h o o l$ of Automation and Information Engineering, Sichuan University of Science and Engineering, Zigong, China. ${ }^{3}$ Department of Radiology, Affiliated Hangzhou First People's Hospital, Zhejiang University School of Medicine, Hangzhou, China. ${ }^{\circledR}$ email: hangzhoudzx73@126.com; jurongding@gmail.com
} 


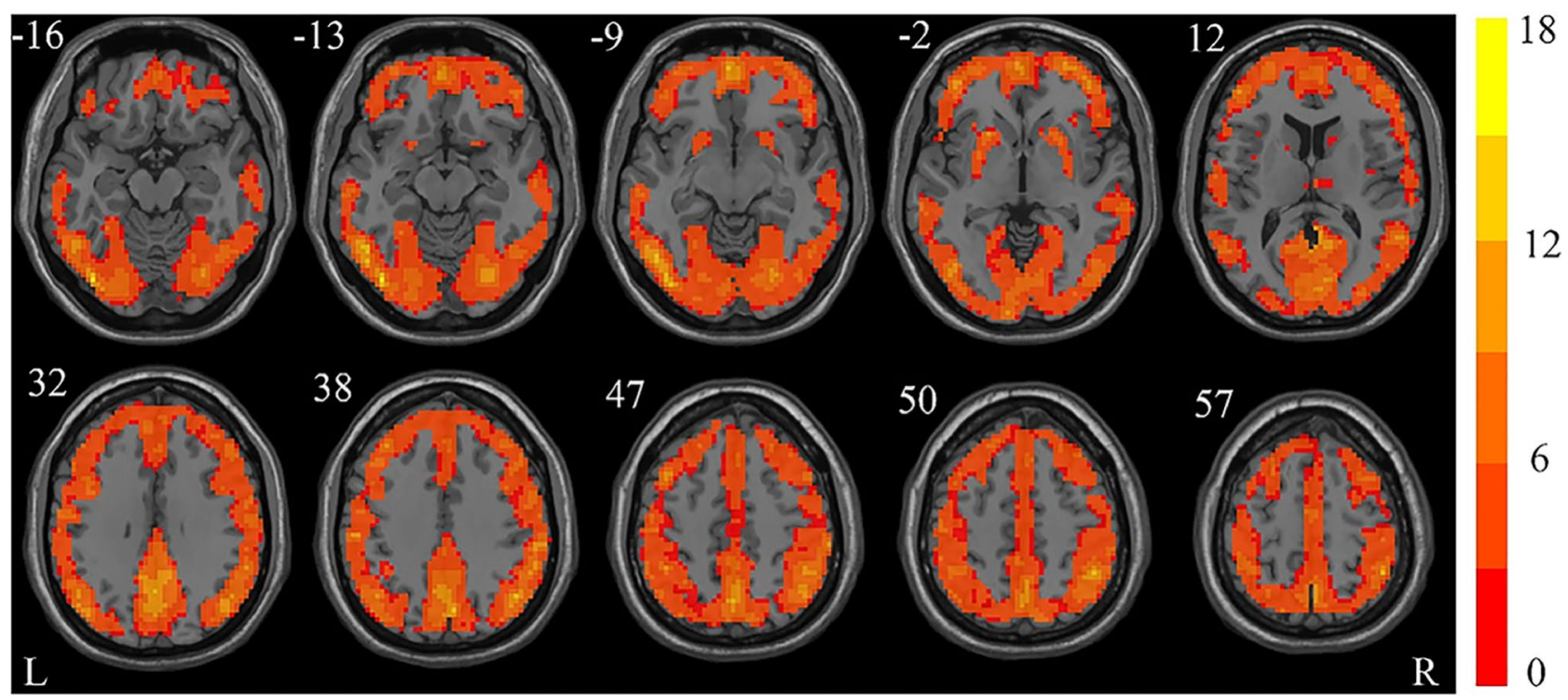

Figure 1. The one-sample $t$-test results of the healthy control group shown as a Kendall's coefficient concordance $(\mathrm{KCC})$ map (false discovery rate $[\mathrm{FDR}]$ corrected, $p<0.05$ ). The figure was presented using the free DPABI software. Red areas indicate a positive ReHo. The number above each image refer to the z-plane coordinates of the Montreal Neurological Institute space. ReHo regional homogeneity, $L$ left, $R$ right.

$\operatorname{cognition}^{19}$. This method has been used in the study of many diseases, including epilepsy ${ }^{20}$, attention-deficit/ hyperactivity disorder ${ }^{21}$, and Alzheimer's disease ${ }^{18,22}$.

Little is known about the changes in spontaneous brain activity, especially the intra-cluster homogeneity of children with GHD in the resting state. In the present study, we performed rs-fMRI and ReHo analysis on children with GHD and HCs. Based on the existing resting-state neuroimaging studies and clinical behavioral studies of children with GHD, we hypothesized that ReHo analysis would identify differences of activity homogeneity in certain clusters between participates with GHD and HCs, especially in brain regions related to social, thought, and behavior problems $s^{5,16}$. In addition, we aimed to explore the difference between the two groups on the brain function and provide imaging data for studying the pathophysiological mechanism of abnormal cognitive and behavioral of children with GHD.

\section{Results}

ReHo for each group. In order to intuitively display ReHo results for the GHD and HC groups, a ReHo map was calculated within each group and graphically displayed in Figs. 1 and 2, respectively (one-sample $t$-test; $p<0.05$, FDR corrected). Through visual observation, it was found that the ReHo values of the bilateral frontal gyrus, supplementary motor area (SMA), cingulate gyrus, cuneus (CUN), occipital gyrus, fusiform gyrus (FFG), parietal gyrus, angular gyrus (ANG), precuneus (PCUN), putamen (PUT) and temporal gyrus were significantly higher than those of other brain regions. This is consistent with previous research results ${ }^{13,23}$. In addition, the bilateral precentral gyrus (PreCG), calcarine fissure and surrounding cortex ${ }^{6}$, lingual gyrus (LING), postcentral gyrus (PoCG) and supramarginal gyrus (SMG) also exhibited higher ReHo values.

Group comparison results. The two-sample $t$-tests results were graphically displayed in Fig. 3 . The statistical significance threshold was set at $p<0.05$, corrected by AlphaSim program (combined a voxel threshold of $p<0.05$ and a minimum cluster size of 56 voxels $)^{24}$. There were several regions showing significant differences in ReHo between the children with GHD and HCs (Table 1). The left PUT showed increased ReHo, while the right PreCG, bilateral orbital parts of the superior frontal gyrus (ORBsup) and left medial part of the superior frontal gyrus (SFGmed) showed decreased ReHo in children with GHD.

\section{Discussion}

To the best of our knowledge, this is the first study to analyze the regional homogeneity of brain activity in children with GHD in a resting state. In the current study, we performed rs-fMRI and ReHo analysis in children with GHD and HCs. Compared to HCs, children with GHD had increased ReHo in the left PUT, and decreased ReHo in the right PreCG, bilateral ORBsup and left SFGmed. These findings indicate that children with GHD have altered brain activity compared to HCs.

Compared with the HCs, the GHD group showed significantly increased ReHo in the left PUT. It has been suggested that this brain region plays a vital role in inhibitory control ${ }^{25}$. Several studies have found that the PUT is more active in more impulsive subjects than in less impulsive subjects ${ }^{26-29}$. Furthermore, in recent fMRI studies, motivated behavior has been shown to be accompanied by a weakened cognitive control of impulses ${ }^{30}$. Consistent with a prior study on $\mathrm{GHD}^{16}$, we also found significantly decreased ReHo in the right PreCG in 

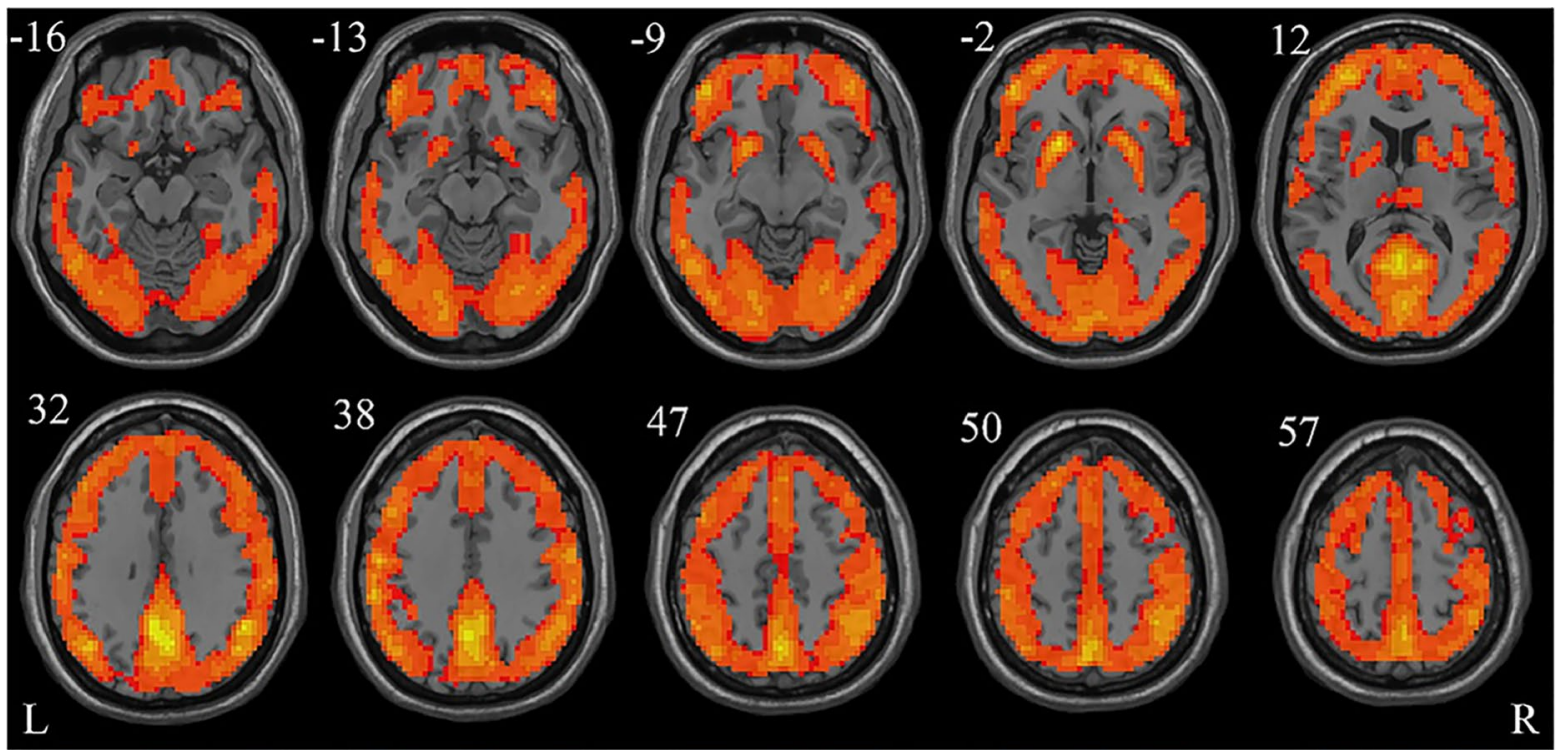

Figure 2. The one-sample $t$-test results of the growth hormone deficiency group shown as a KCC map (FDR corrected, $p<0.05)$. The figure was presented using the free DPABI software. Red areas indicate a positive ReHo. The number above each image refer to the z-plane coordinates of the Montreal Neurological Institute space. ReHo regional homogeneity, $L$ left, $R$ right.

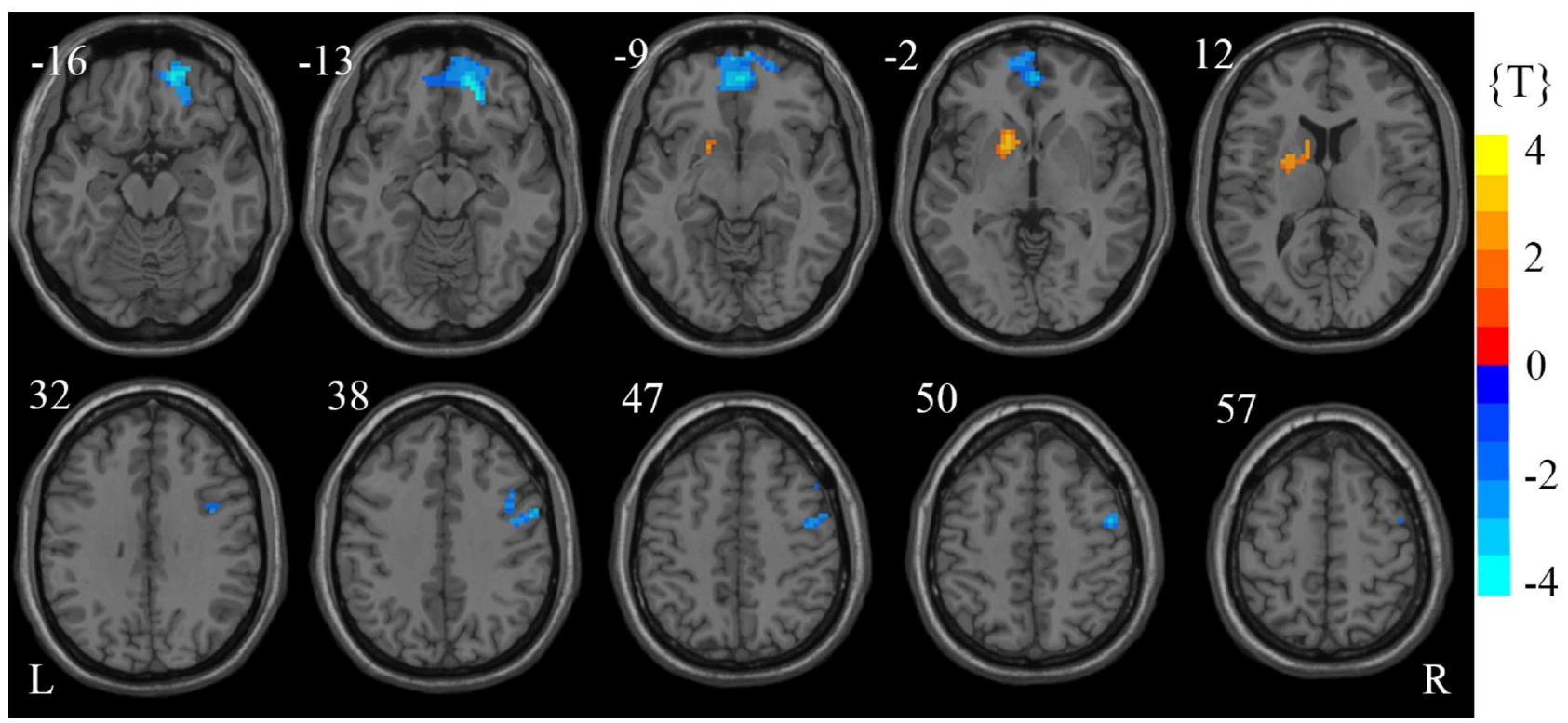

Figure 3. Statistically significant differences in regional homogeneity (ReHo) between children with GHD and healthy controls ( $p<0.05$, AlphaSim corrected). The figure was presented using the free DPABI software. Cold and hot colors in the color bar indicate decreased and increased ReHo, respectively, in children with GHD. The number above each image refer to the z-plane coordinates of the Montreal Neurological Institute space. GHD growth hormone deficiency, $L$ left, $R$ right.

children with GHD. The PreCG is located in the premotor cortex and is associated with the somatic sensorimotor network ${ }^{31-33}$. Rech et al. found that the white matter underneath the premotor cortex could immediately elicit a unilateral negative motor response (UNMR) when stimulated ${ }^{34}$. Meanwhile, Schucht et al. suggested that the neural network causing UNMRs may be related to motor control ${ }^{35}$. Thus, we speculate that the GHD group's increased ReHo in the left PUT and decreased ReHo in the right PreCG may be associated with motor control dysfunction, which may reflect the pathophysiological mechanism of impulsive and hyperactive characteristics of behavior in children with GHD GH,36,37. $^{\text {. }}$

In the current study, we also observed decreased ReHo in children with GHD in bilateral ORBsup, a region that is located in the medial orbitofrontal cortex (mOFC). The ORBsup has been shown to play a vital role in choosing the right response and suppressing the wrong response ${ }^{38,39}$. In a previous study, patients with 


\begin{tabular}{|l|l|l|l|l|}
\hline Brain region & Voxels & MNI (x,y,z) & B & BA \\
\hline ReHo increased regions & \multicolumn{5}{|l|}{} \\
\hline PUT.L & 40 & $-15,12,-3$ & 2.8651 & 48 \\
\hline ReHo decreased regions & 66 & $54,0,42$ & -3.3343 & 6 \\
\hline PreCG.R & 52 & $21,42,-12$ & -4.0408 & 11 \\
\hline ORBsup.R & 105 & $0,51,-6$ & -3.4678 & $10 / 11$ \\
\hline ORBsupmed.L & 59 & $3,51,-6$ & -3.4439 & $10 / 11$ \\
\hline ORBsupmed.R & 49 & $0,36,57$ & -3.1438 & $8 / 32$ \\
\hline SFGmed.L & &
\end{tabular}

Table 1. Regions of increased/decreased ReHo in children with GHD. ReHo regional homogeneity, GHD growth hormone deficiency, MNI $(x, y, z)$ coordinates of the peak voxel in the MNI space, $t$ the statistical value of peak voxel, with a positive $t$-value indicating an increased ReHo and a negative $t$-value indicating a decreased ReHo in patients with GHD, BA Brodmann's area, $L$ left, $R$ right, $P U T$ putamen, PreCG precentral gyrus, ORBsup superior frontal gyrus, orbital part, ORBsupmed superior frontal gyrus, medial orbital, SFGmed superior frontal gyrus, medial.

ORBsup lesions were found to have behavioral regulation failures in the absence of external stimuli ${ }^{38}$. Decreased mOFC activity can be detected when patients with anxiety and depression fail to inhibit inappropriate anxiety responses ${ }^{40-42}$. In addition, decreased ReHo was also observed in the SFGmed, located in the medial prefrontal cortex (MPFC). The MPFC is one of the most important functional areas in the default mode network ${ }^{43}$, and is considered to participate in emotional processing, such as monitoring anxiety and other psychological states ${ }^{4-47}$. The significantly decreased ReHo in the MPFC of anxiety patients was linked to a decrease in the ability to regulate emotion ${ }^{13}$. In the present study, the decreased ReHo of the ORBsup and SFGmed in the GHD group may be associated with inactivation of emotional regulation, which may underlie the anxiety and depression observed in children with $\mathrm{GHD}^{5,36,37,41}$.

Several limitations of this study need to be addressed. Firstly, the number of subjects included in the study was small, especially in the HC group. Therefore, the generalizability of this study to the whole GHD population is limited. However, since both gender and age were matched between the two groups, we believe this constraint did not affect the robustness of our results. Secondly, participants in this study were not assessed by clinical cognitive and behavioral scales. It is valuable to evaluate the relationship of neuroimaging results with clinical scales. Therefore, in future studies, assessments of relevant scales should be included.

\section{Methods}

Participants and ethical considerations. All subjects were recruited from the pediatric clinic of the Zhejiang Provincial People's Hospital from October 2016 to March 2018. All participants' parents gave written informed consent for their inclusion in the study, and this study was approved by the Medical Ethics Committee of Zhejiang Provincial People's Hospital and adhered to the tenets of the Declaration of Helsinki. All subjects were right-handed and prepubescent. Participants were excluded if they met the following criteria: (1) head movements were obviously too large during MRI scans; (2) abnormalities identified on MRI associated with GHD; (3) had congenital multiple pituitary hormone deficiency (MPHD) or thyroid axis hormone level abnormalities; (4) chronic liver, kidney, or skeletal system diseases; (5) chromosomal abnormalities or special facial/ abnormal signs with diagnostic significance; (6) hyperthermia during the scanning; (7) past medical history of seizures, benign masses or cancers, or psychological disease/psychosis; (8) family history of mental retardation or congenital heart disease, or (9) contraindications to MRI (such as metal implants or claustrophobia).

Growth hormone $(\mathrm{GH})$ stimulation tests were performed in participants with short stature. Because of the limitations of GH stimulation tests, GHD can only be diagnosed when the results of two or more drug stimulation tests are abnormal ${ }^{1,48}$. Seven children with short stature were excluded due to their peak GH value, which was higher than $10 \mu \mathrm{g} / \mathrm{L}$, during the GH stimulation test. Accordingly, the first group was composed of 26 children with GHD (11 males, mean age: $8.2 \pm 1.9$ years), in whom both arginine and clonidine GH stimulation tests showed a partial or complete lack of GH $(<10 \mu \mathrm{g} / \mathrm{L})^{1}$. Furthermore, 15 age- and sex-matched HCs formed the second group (7 males, mean age: $8.6 \pm 1.7$ years), who had normal height and normal growth variation. There were no statistically significant differences in age $(p=0.3823$, two-tailed Mann-Whitney $U$ test $)$ or $\operatorname{sex}(p>0.9999$, two-tailed Fisher's exact test) between the two groups.

Imaging data acquisition. fMRI scans were performed with a 3.0-T MRI scanner (Discovery MR750; GE Healthcare, Milwaukee, WI) prior to GH stimulation tests in all participants during the resting state to avoid the effects of stimulating drugs. The subjects were instructed to lie as still as possible but not fall asleep. Two foam pads were used on the head to reduce head movement, and earplugs were worn to reduce external noise. The MRI equipment and data parameters used in all MR imaging examinations were consistent with our previous study ${ }^{49}$. The specific parameter settings were as follows: repetition time $=2000 \mathrm{~ms}$, echo time $=30 \mathrm{~ms}$, flip angle $=90^{\circ}$, field of view $=220 \mathrm{~mm}$, matrix size $=64 \times 64$, voxel size $=3.44 \times 3.44 \times 3.2 \mathrm{~mm}^{3}$, no slice gap and 44 axial slices. The first 10 volumes of all acquired rs-fMRI data image sequences were excluded in order to ensure that the subjects were stable in the resting state. 
Data preprocessing. The rs-fMRI data processing was performed using DPABI software (Version V4.0_190305, http://rfmri.org/dpabi $)^{50}$, followed by temporal difference and head-motion corrections on the remaining 200 volumes. No participants were excluded for translation or rotation parameters exceeding $3.0 \mathrm{~mm}$ or $3.0^{\circ}$ of head motion. Previous studies have reported that rs-fMRI findings is sensitive to head motion effects $^{51,52}$. Here, we also computed the frame-wise displacement (FD) index ${ }^{51}$, and found no significant differences $(p=0.7175)$ in head movement between children with GHD $(0.0936 \pm 0.0632)$ and HCs $(0.1009 \pm 0.0584)$ using two-sample $t$-tests. The echo-planar imaging (EPI) template was used to spatially normalize the image into the Montreal Neurological Institute (MNI) space and resampled to $3 \times 3 \times 3 \mathrm{~mm}^{3}$ voxels. Subsequently, to remove the effects of head motion and other possible sources of artifacts, we regressed the constant, linear trend, and head motion parameters (Friston 24-parameter model) ${ }^{53}$ as covariates ${ }^{54}$. The time series signals of the cerebrospinal fluid and white matter were eliminated by using the prior template in SPM8 software (Statistical Parametric Mapping 8, http://www.fil.ion.ucl.ac.uk/spm/). Finally, a band-pass filter $(0.01<f<0.08 \mathrm{~Hz})$ was used to remove the effects of noise, such as respiratory rhythm.

ReHo analysis. For the preprocessed rs-fMRI data, the time series of specific voxels were extracted. We defined the 27 nearest voxels as a cluster and calculated their ReHo. The Kendall's coefficient concordance (KCC) values measure the consistency of activities between adjacent voxels and are assigned to a given voxel ${ }^{17}$, according to the following Eq. (1):

$$
W=\frac{\sum\left(R_{i}\right)^{2}-n(\bar{R})^{2}}{\frac{1}{12} K^{2}\left(n^{3}-n\right)}
$$

where $W$ was the KCC among given voxels, ranging from 0 to $1 ; R_{i}$ was the sum rank of the $i$ th time point; $\bar{R}=((n+1) K) / 2$ was the mean of the $R_{i}$ s; $K$ was the number of time series within a measured cluster (here $K=27$ ); and $n$ was the number of ranks (here $n=200$ ). The individual KCC map was generated with the free DPABI software (Version V4.0_190305, http://rfmri.org/dpabi) ${ }^{50}$. Finally, spatial smoothing was performed in KCC maps with a full width at half maximum (FWHM) of $4 \mathrm{~mm}$ to decrease spatial noise.

Statistical analysis. Second-level analyses of KCC maps were carried out using the SPM 8 software package. The brain regions with a KCC value greater than 1 were detected by performing one-sample t-tests within the two groups. A mask was used, according to the automated anatomical labeling (AAL) templates ${ }^{55}$, which matched the size of the spatially normalized voxels $\left(3 \times 3 \times 3 \mathrm{~mm}^{3}\right)$. The multiple comparisons were performed using the false discovery rate (FDR) criterion $(p<0.05)^{56}$.

The one-sample $t$-tests results from the two groups were combined to get a new map. By binarizing the map, a combined explicit mask was obtained. Then, two-sample $t$-tests were performed within this mask to compare the ReHo between two groups. The results of the two-sample $t$-tests were corrected by using the AlphaSim program in the RESTplus software (Resting-State fMRI Data Analysis Toolkit plus V1.24, http://www.restfmri.net/forum/ RESTplus $)^{24}$, as determined by a Monte Carlo simulation to calculate the probability of false-positive detection ${ }^{13}$. The AlphaSim correction was confined within the combined mask of one-sample t-tests results from the two groups, and the size of smooth kernel was set at $4 \mathrm{~mm}$. The threshold of the $t$-map was set at a combined height threshold of $p<0.05$ and a minimum cluster size of 56 voxels, and this corresponded to a corrected $p<0.05$.

\section{Conclusions}

In the current study, we performed fMRI and ReHo calculations in children with GHD and HCs in the resting state. Our findings of increased ReHo in the left PUT and decreased ReHo in the right PreCG may suggest a dysfunction of inhibitory control in children with GHD. In addition, the decreased ReHo was observed in the bilateral ORBsup and left SFGmed, reflecting a possible inactivation of the inhibition of inappropriate anxiety and depression in children with GHD. In summary, the present findings confirmed that the activity homogeneity in specific brain regions is altered in children with GHD, providing imaging evidence to elucidate potential pathophysiological mechanisms of abnormal cognition and behavior in children with GHD.

Received: 29 July 2020; Accepted: 9 December 2020

Published online: 11 January 2021

\section{References}

1. Society, G. R. Consensus guidelines for the diagnosis and treatment of growth hormone (GH) deficiency in childhood and adolescence: summary statement of the GH research society. J. Clin. Endocrinol. Metab. 85, 3990-3993. https://doi.org/10.1210/ jcem.85.11.6984 (2000).

2. Wang, Q., Liu, D. Y., Yang, L. Q., Liu, Y. \& Chen, X. J. The epidemic characteristics of short stature in school students. Ital. J. Pediatr. 41, 99. https://doi.org/10.1186/s13052-015-0207-6 (2015).

3. Morrison, M. Valuing height: diagnosis, valuation and the case of idiopathic short stature. Sociol. Health Illn 41, 502-516. https:// doi.org/10.1111/1467-9566.12828 (2019).

4. Kyung, C. S. et al. Etiologies and characteristics of children with chief complaint of short stature. Ann. Pediatr. Endocrinol. Metab. 20, 34-39. https://doi.org/10.6065/apem.2015.20.1.34 (2015).

5. Stabler, B. et al. Behavior change after growth hormone treatment of children with short stature. J. Pediatr. 133, 366-373. https:// doi.org/10.1016/S0022-3476(98)70271-9 (1998).

6. Bozzola, M. et al. Role of magnetic resonance imaging in the diagnosis and prognosis of growth hormone deficiency. Clin. Endocrinol. 45, 21-26. https://doi.org/10.1111/j.1365-2265.1996.tb02055.x (1996).

7. Vannelli, S. et al. Magnetic resonance and the diagnosis of short stature of hypothalamic-hypophyseal origin. Acta Paediatr. 82, 155-161. https://doi.org/10.1111/j.1651-2227.1993.tb12629.x (1993). 
8. Hamilton, J. et al. Familial growth hormone deficiency associated with MRI abnormalities. Am. J. Med. Genet. 80, 128-132. https ://doi.org/10.1002/(SICI) 1096-8628(19981102)80:2\%3c128::AID-AJMG7\%3e3.0.CO;2-C (1998).

9. D'Esposito, M., Deouell, L. Y. \& Gazzaley, A. Alterations in the BOLD fMRI signal with ageing and disease: a challenge for neuroimaging. Nat. Rev. Neurosci. 4, 863-872. https://doi.org/10.1038/nrn1246 (2003).

10. Kwong, K. K. et al. Dynamic magnetic resonance imaging of human brain activity during primary sensory stimulation. Proc. Natl. Acad. Sci. USA 89, 5675-5679. https://doi.org/10.1073/pnas.89.12.5675 (1992).

11. Ogawa, S., Lee, T. M., Nayak, A. S. \& Glynn, P. Oxygenation-sensitive contrast in magnetic resonance image of rodent brain at high magnetic fields. Magn. Reson. Med. 14, 68-78. https://doi.org/10.1002/mrm.1910140108 (1990).

12. Tomasi, D. \& Volkow, N. D. Abnormal functional connectivity in children with attention-deficit/hyperactivity disorder. Biol. Psychiat. 71, 443-450. https://doi.org/10.1016/j.biopsych.2011.11.003 (2011).

13. Qiu, C. et al. Regional homogeneity changes in social anxiety disorder: A resting-state fMRI study. Psychiatry Res. Neuroimaging 194, 47-53. https://doi.org/10.1016/j.pscychresns.2011.01.010 (2011).

14. Roger, E. et al. Hubs disruption in mesial temporal lobe epilepsy. A resting-state fMRI study on a language-and-memory network. Human Brain Mapping 41, 779-796. https://doi.org/https://doi.org/10.1002/hbm.24839 (2020).

15. Li, G. et al. Large-scale dynamic causal modeling of major depressive disorder based on resting-state functional magnetic resonance imaging. Hum. Brain Mapp. 41, 865-881. https://doi.org/10.1002/hbm.24845 (2020).

16. Hu, Y. et al. Differences in the functional connectivity density of the brain between individuals with growth hormone deficiency and idiopathic short stature. Psychoneuroendocrinology 103, 67-75. https://doi.org/10.1016/j.psyneuen.2018.12.229 (2019).

17. Zang, Y., Jiang, T., Lu, Y., He, Y. \& Tian, L. Regional homogeneity approach to fMRI data analysis. Neuroimage 22, 394-400. https ://doi.org/10.1016/j.neuroimage.2003.12.030 (2004).

18. He, Y. et al. Regional coherence changes in the early stages of Alzheimer's disease: A combined structural and resting-state functional MRI study. Neuroimage 35, 488-500. https://doi.org/10.1016/j.neuroimage.2006.11.042 (2007).

19. Liu, Z. et al. Decreased regional homogeneity in insula and cerebellum: a resting-state fMRI study in patients with major depression and subjects at high risk for major depression. Psychiatry Res. Neuroimaging 182, 211-215. https://doi.org/10.1016/j.pscyc hresns.2010.03.004 (2010).

20. Zeng, H. et al. Regional homogeneity ( $\mathrm{ReHo}$ ) changes in new onset versus chronic benign epilepsy of childhood with centrotemporal spikes (BECTS): A resting state fMRI study. Epilepsy Res. 116, 79-85. https://doi.org/10.1016/j.eplepsyres.2015.06.017 (2015).

21. Zhu, C. et al. Fisher discriminative analysis of resting-state brain function for attention-deficit/hyperactivity disorder. Neuroimage 40, 110-120. https://doi.org/10.1016/j.neuroimage.2007.11.029 (2008).

22. Liu, Y. et al. Regional homogeneity, functional connectivity and imaging markers of Alzheimer's disease: a review of resting-state fMRI studies. Neuropsychologia 46, 1648-1656. https://doi.org/10.1016/j.neuropsychologia.2008.01.027 (2008).

23. Ke, J. et al. Abnormal regional homogeneity in patients with irritable bowel syndrome: A resting-state functional MRI study. Neurogastroenterol. Motil. 27, 1796-1803. https://doi.org/10.1111/nmo.12692 (2015).

24. Jia, X.-Z. et al. RESTplus: an improved toolkit for resting-state functional magnetic resonance imaging data processing. Sci. Bull. 64, 953-954. https://doi.org/10.1016/j.scib.2019.05.008 (2019).

25. Akkermans, S. E. A., Luijten, M., van Rooij, D., Franken, I. H. A. \& Buitelaar, J. K. Putamen functional connectivity during inhibitory control in smokers and non-smokers. Addict. Biol. 23, 359-368. https://doi.org/10.1111/adb.12482 (2018).

26. Horn, N. R., Dolan, M., Elliott, R., Deakin, J. F. W. \& Woodruff, P. W. R. Response inhibition and impulsivity: an fMRI study. Neuropsychologia 41, 1959-1966. https://doi.org/10.1016/S0028-3932(03)00077-0 (2003).

27. Collins, H. R. et al. Too little, too late or too much, too early? Differential hemodynamics of response inhibition in high and low sensation seekers. Brain Res. 1481, 1-12. https://doi.org/10.1016/j.brainres.2012.08.004 (2012).

28. Chester, D. S. \& DeWall, C. N. Prefrontal recruitment during social rejection predicts greater subsequent self-regulatory imbalance and impairment: neural and longitudinal evidence. NeuroImage 101, 485-493. https://doi.org/10.1016/j.neuroimage.2014.07.054 (2014).

29. Schmüser, L. et al. Data-driven analysis of simultaneous EEG/fMRI reveals neurophysiological phenotypes of impulse control. Hum. Brain Mapp. 37, 3114-3136. https://doi.org/10.1002/hbm.23230 (2016).

30. Zhukovsky, P., Morein-Zamir, S., Meng, C., Dalley, J. W. \& Ersche, K. D. Network failures: When incentives trigger impulsive responses. Hum. Brain Mapp. 41, 2216-2228. https://doi.org/10.1002/hbm.24941 (2020).

31. Beckmann, C. F., Marilena, D. L., Devlin, J. T. \& Smith, S. M. Investigations into resting-state connectivity using independent component analysis. Philos. Trans. R Soc. Lond. B Biol. Sci. 360, 1001-1013. https://doi.org/10.1098/rstb.2005.1634 (2005).

32. Luca, M. D., Beckmann, C. F., Stefano, N. \& De, M. fMRI resting state networks define distinct modes of long-distance interactions in the human brain. Neuroimage 29, 1359-1367. https://doi.org/10.1016/j.neuroimage.2005.08.035 (2006).

33. de Bie, H. M. et al. Resting-state networks in awake five- to eight-year old children. Human Brain Mapping 33, 1189-1201. https:// doi.org/https://doi.org/10.1002/hbm.21280 (2012).

34. Rech, F., Herbet, G., Moritz-Gasser, S. \& Duffau, H. Disruption of bimanual movement by unilateral subcortical electrostimulation. Hum. Brain Mapp. 35, 3439-3445. https://doi.org/10.1002/hbm.22413 (2014).

35. Schucht, P., Moritz-Gasser, S., Herbet, G., Raabe, A. \& Duffau, H. Subcortical electrostimulation to identify network subserving motor control. Hum. Brain Mapp. 34, 3023-3030. https://doi.org/10.1002/hbm.22122 (2013).

36. Stabler, B., Tancer, M. E., Ranc, J. \& Underwood, L. E. Evidence for social phobia and other psychiatric disorders in adults who were growth hormone deficient during childhood. Anxiety 2, 86-89. https://doi.org/10.1002/(SICI)1522-7154(1996)2:2\%3c86::AIDANXI4\%3e3.0.CO;2-B (1996).

37. Stabler, B. et al. Academic achievement and psychological adjustment in short children. J. Dev. Behav. Pediatr. 15, 1-6. https://doi. org/10.1097/00004703-199402000-00001 (1994).

38. Goldman-Rakic, P. S. Circuitry of primate prefrontal cortex and regulation of behavior by representational memory. (Comprehensive Physiology, 2011).

39. Robbins, T. W. The prefrontal cortex, 2nd edn. J. M. Fuster. Raven Press, New York, 1989. Int. J. Geriatric Psychiatry 5, 348-348. https://doi.org/10.1002/gps.930050518 (1990).

40. Milad, M. R. \& Rauch, S. L. The role of the orbitofrontal cortex in anxiety disorders. Ann. N. Y. Acad. Sci. 1121, 546-561. https:// doi.org/10.1196/annals.1401.006 (2007).

41. Drevets, W. C. Orbitofrontal cortex function and structure in depression. Ann. N. Y. Acad. Sci. 1121, 499-527. https://doi. org/10.1196/annals.1401.029 (2007).

42. Rempel-Clower, N. L. Role of orbitofrontal cortex connections in emotion. Ann. N. Y. Acad. Sci. 1121, 72-86. https://doi. org/10.1196/annals.1401.026 (2007).

43. Raichle, M. E. et al. A default mode of brain function. Proc. Natl. Acad. Sci. 98, 676-682. https://doi.org/10.1073/pnas.98.2.676 (2001).

44. Blakemore, S. J. The social brain in adolescence. Nat. Rev. Neurosci. 9, 267-277. https://doi.org/10.1038/nrn2353 (2008).

45. Gusnard, D. A. \& Raichle, M. E. Searching for a baseline: Functional imaging and the resting human brain. Nat. Rev. Neurosci. 2, 685-694. https://doi.org/10.1038/35094500 (2001).

46. Kelley, W. M. et al. Finding the self? An event-related fMRI study. J. Cognit. Neurosci. 14, 785-794. https://doi.org/10.1162/08989 $290260138672(2002)$. 
47. Fossati, P. et al. Distributed self in episodic memory: neural correlates of successful retrieval of self-encoded positive and negative personality traits. Neuroimage 22, 1596-1604. https://doi.org/10.1016/j.neuroimage.2004.03.034 (2004).

48. Chesover, A. D. \& Dattani, M. T. Evaluation of growth hormone stimulation testing in children. Clin. Endocrinol. 84, 708-714. https://doi.org/10.1111/cen.13035 (2016).

49. Ding, J.-R. et al. Presurgical localization and spatial shift of resting state networks in patients with brain metastases. Brain Imaging Behav. 13, 408-420. https://doi.org/10.1007/s11682-018-9864-6 (2019).

50. Yan, C. G., Wang, X. D., Zuo, X. N. \& Zang, Y. F. DPABI: Data processing and analysis for (resting-state) brain imaging. Neuroinformatics 14, 339-351. https://doi.org/10.1007/s12021-016-9299-4 (2016).

51. Jenkinson, M., Bannister, P., Brady, M. \& Smith, S. Improved optimization for the robust and accurate linear registration and motion correction of brain images. NeuroImage 17, 825-841. https://doi.org/10.1006/nimg.2002.1132 (2002).

52. Power, J. D., Barnes, K. A., Snyder, A. Z., Schlaggar, B. L. \& Petersen, S. E. Spurious but systematic correlations in functional connectivity MRI networks arise from subject motion. NeuroImage 59, 2142-2154. https://doi.org/10.1016/j.neuroimage.2011.10.018 (2012).

53. Friston, K. J., Williams, S., Howard, R., Frackowiak, R. S. J. \& Turner, R. Movement-related effects in fMRI time-series. Magn. Reson. Med. 35, 346-355. https://doi.org/10.1002/mrm.1910350312 (1996).

54. Fox, M. D. et al. The human brain is intrinsically organized into dynamic, anticorrelated functional networks. Proc. Natl. Acad. Sci. USA 102, 9673-9678. https://doi.org/10.1073/pnas.0504136102 (2005).

55. Tzourio-Mazoyer, N. et al. Automated anatomical labeling of activations in SPM using a macroscopic anatomical parcellation of the MNI MRI single-subject brain. Neuroimage 15, 273-289. https://doi.org/10.1006/nimg.2001.0978 (2002).

56. Genovese, C. R., Lazar, N. A. \& Nichols, T. Thresholding of statistical maps in functional neuroimaging using the false discovery rate. Neuroimage 15, 870-878. https://doi.org/10.1006/nimg.2001.1037 (2002).

\section{Acknowledgements}

This work was supported by the National Natural Science Foundation of China (Grant numbers 81401482, 81871337); the Research Foundation of Artificial Intelligence Key Laboratory of Sichuan Province (Grant number 2019RYJ04); the Scientific Research Foundation of Sichuan University of Science and Engineering (Grant number 2020RC31); and the Innovation Fund of Postgraduate, Sichuan University of Science and Engineering (Grant number y2019020). We thank all the subjects for participating in this study.

\section{Author contributions}

Z.D. and J.D. contributed to the design of the study. M.W. and Z.D. collected the data. F.Z., B.H. and T.W. analyzed the data and prepared the figures. F.Z., M.W. and J.D. prepared the original draft. F.Z., Z.D. and J.D. revised the manuscript with input from all authors, and acquired the funding.

\section{Competing interests}

The authors declare no competing interests.

\section{Additional information}

Correspondence and requests for materials should be addressed to Z.D. or J.-R.D.

Reprints and permissions information is available at www.nature.com/reprints.

Publisher's note Springer Nature remains neutral with regard to jurisdictional claims in published maps and institutional affiliations.

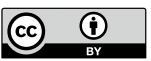

Open Access This article is licensed under a Creative Commons Attribution 4.0 International License, which permits use, sharing, adaptation, distribution and reproduction in any medium or format, as long as you give appropriate credit to the original author(s) and the source, provide a link to the Creative Commons licence, and indicate if changes were made. The images or other third party material in this article are included in the article's Creative Commons licence, unless indicated otherwise in a credit line to the material. If material is not included in the article's Creative Commons licence and your intended use is not permitted by statutory regulation or exceeds the permitted use, you will need to obtain permission directly from the copyright holder. To view a copy of this licence, visit http://creativecommons.org/licenses/by/4.0/.

(C) The Author(s) 2021 